\title{
A simple quantitative and qualitative microbiological screening test for bacteriuria
}

\author{
E. M. MACKAY-SCOLLAY
}

From the Public Health Laboratory Service, Western Australia

SYNOPSIS A simple, accurate screening test for significant bacteriuria is described. It entails brief contact of a freshly voided specimen of urine with the surface of a solid culture medium lining the wall of a transparent disposable plastic container, incubating it at $37^{\circ} \mathrm{C}$ for 24 hours, and counting colonies developing on a proscribed surface area. The method obviates errors due to contaminants, affords a limited species differentiation of the causative organisms, and provides a ready means of obtaining a direct sensitivity test on the responsible bacteria.

The diagnosis of a urinary tract infection depends upon the demonstration of the responsible microorganisms in one or more specimens of urine from the patient. However, the picture is frequently obscured by the presence of contaminating organisms from the urethra and vulva which can multiply in the urine, which is an excellent culture medium (Asscher, Sussman, Waters, Harvard Davis, and Chick, 1966), between the time of collection and microbiological examination. Refrigeration of the specimen, for a limited period immediately after voiding, partly overcomes the multiplication of contaminants and allows the significance of bacteriuria to be determined by the enumeration of 100,000 or more organisms per millilitre of urine (Kass, 1956). However, it is not always convenient or possible to refrigerate urine, and the time of transit of specimens between the clinic and the laboratory can be considerable, thus providing erroneous readings in viable counts.

Methods employed in the laboratory to enumerate organisms in urine specimens are principally modifications of techniques used for performing total viable counts (Cattell and Lefford, 1963; Hoeprich, 1960; Mackie and McCartney, 1960). These demand considerable time in their performance and skill in their interpretation. Relatively simple microbiological tests have been devised by Leigh and Williams (1964), Mackey and Sandys (1965), and by Naylor and Guttmann (1967) but these have disadvantages either of inaccuracy or inconvenience. A need exists for a simple and accurate method of determining significant bacteriuria (Lancet, 1968), not only

Received for publication 13 February 1969. to confirm clinical evidence of urinary tract infection but to enable population surveys to be carried out to uncover inapparent or subclinical infections which frequently lead to irreversible renal damage.

A simple and reproducible method of performing total viable counts within the range $10^{5}$ to $10^{4}$ organisms per millilitre on freshly voided urine samples is reported.

\section{MATERIALS AND METHODS}

Suspensions of microorganisms in urine were serially diluted in autoclaved urine and these dilutions were poured into clear, sterile polystyrene containers lined by nutrient agar medium.

MEDIUM A simple medium was used throughout. (All quantities are in $\mathrm{g} /$ litre.)

\begin{tabular}{|c|c|c|c|c|c|c|c|}
\hline Peptone (Oxoid) & . & . & $\cdots$ & . & $\ldots$ & $\ldots$ & $20 \cdot 00$ \\
\hline Lactose & $\ldots$ & . & $\cdots$ & $\cdots$ & $\ldots$ & $\cdots$ & 10.00 \\
\hline Sodium chloride & . & . & . & . & $\ldots$ & . & $2 \cdot 50$ \\
\hline Urea $\quad$. & . & .. & .. & . & $\ldots$ & $\ldots$ & 5.00 \\
\hline Neutral red . . & . & .. & . & . & $\ldots$ & . & 0.5 \\
\hline Agar (Davis) .. & $\ldots$ & $\ldots$ & . & $\ldots$ & $\ldots$ & $\ldots$ & 20.00 \\
\hline
\end{tabular}

The $p \mathrm{H}$ of the medium was adjusted to approximately $7 \cdot 2$ and autoclaved at $10 \mathrm{lb}$ for 10 minutes.

The lining was achieved initially by pouring excess molten medium into the container, rolling it and then pouring off excess of medium before allowing the agar to set.

Later the culture tubes were prepared commercially. The suspensions were left only momentarily in the containers before discarding. Excess fluid was drained off for a few seconds and, after securing the screw caps of the containers, the complete outfits, termed 'rinse cultures', were incubated in an inverted position for 24 hours at $37^{\circ} \mathrm{C}$. 


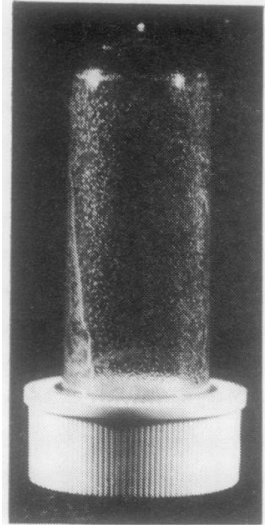

FIG. 1a.

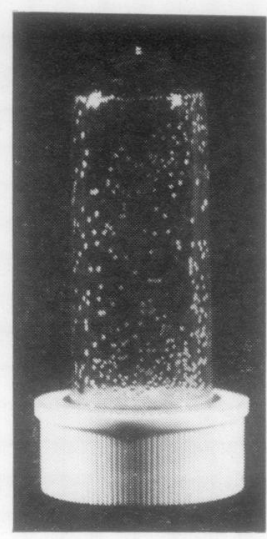

FIG, 1b.

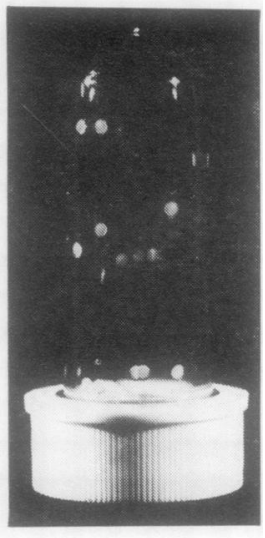

FIG. 1c.
FIG. 1. Rinse cultures of dilutions of a suspension of $\mathrm{E}$. coli (a) $10^{-1}$, (b) $10^{-3}$, and (c) $10^{-5}$.
RESULTS

Colonies appearing on the surface of the medium bore the same quantitative relationship, for a given surface area, to the number of microorganisms present in the suspension as determined by parallel viable counts using standard methods (Mackie and McCartney, 1960). Figure 1 shows colonies developed from serial 10-fold dilutions of Escherichia coli in rinse culture tubes.

The number of colonies developing was counted in the area demarcated on a cellophane sleeve which was capable of being positioned over any given area of the culture tube (Fig. $2 a$ and $2 b$ ). It was found $z$ that within the circle area of $0.23 \mathrm{sq}$ in $(1.43 \mathrm{sq} \mathrm{cm}) \lesssim$ a count of 50 to 80 colonies represented a concen- 3

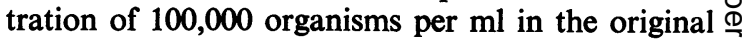
suspension, 25 to 40 colonies of 50,000 , and five to 10 colonies of 10,000 organisms per millilitre.

Subsequently, clinical specimens of urine were examined both by the rinse culture method and by routine standard viable count procedures (Mackie and McCartney, 1960) to determine significant bacteriuria. Close agreement was achieved between the methods. It was found that with practice it was unnecessary actually to count colonies, and reference

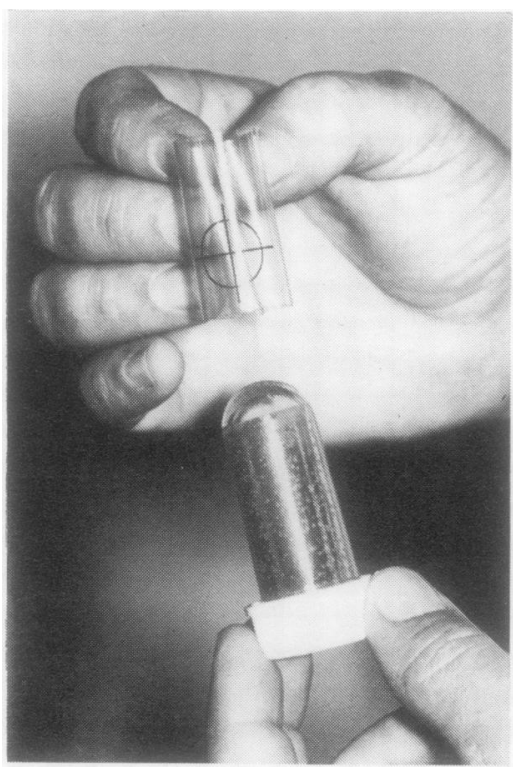

FIG. 2a.

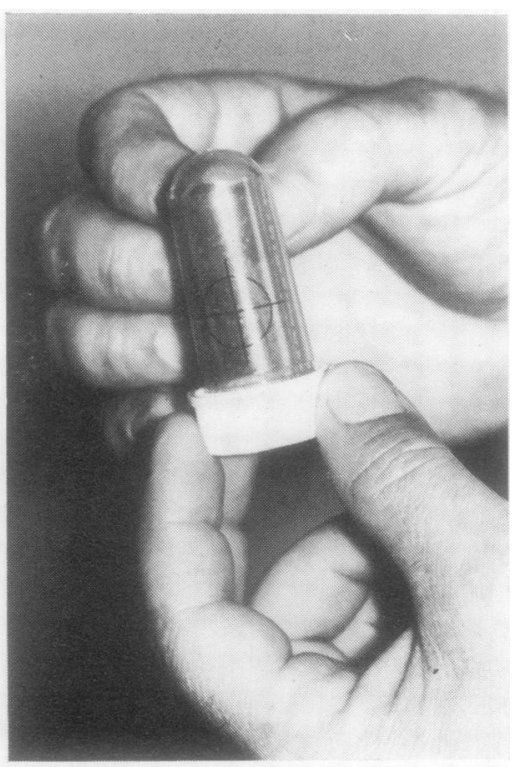

FIG. $2 b$.
FIG. 2. The use of $a$ cellophane sleeve on which is marked the area for making the colony counts. 


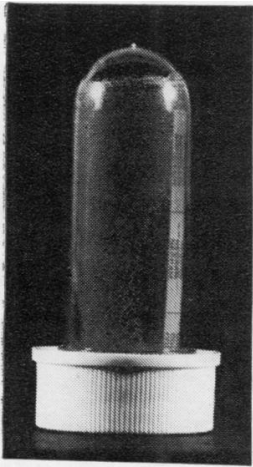

FIG. 3. Rinse culture showing direct antibiotic sensitivity test.

to prepared standard tubes was sufficiently accurate.

The medium used in the rinse cultures provided colour changes relative to the specific microorganisms found in clinical specimens of urine if these were present in pure or almost pure culture.

A refinement of the method was effected by the use of previously prepared strips of sterilized absorbent paper impregnated, on separate sites, with up to three antibiotics, and placing one longitudinally on the surface of the medium inside the culture tube at the time of inoculation, thus achieving a direct antibiotic sensitivity test on the culture organisms, as shown in Figure 3.

\section{DISCUSSION}

The method described for the determination of significant bacteriuria has distinct advantages; it is simple to perform, requiring only the pouring of a sample of the urine specimen into a culture tube lined with a suitable solid medium, of pouring the sample out again, of incubating at $37^{\circ} \mathrm{C}$ overnight followed by a simple counting procedure of the colonies developing under a prescribed surface area of the tube. The procedure may be carried out by relatively untrained persons immediately after voiding of the urine. The unit, once inoculated, may be readily transported to the laboratory for incubation and determination of the significance of the viable count obtained. The accuracy of the counts compares favourably with those made by parallel testing of samples by standard techniques.
The use of the unit has valuable applications in domiciliary practice as well as in ward or clinic. The immediacy of the method of inoculating the culture ensures that the viable count represents bacteria actually present in the urine sample at the time of passing, thereby eliminating errors due to contaminating organisms. Specimens collected at a distance from the laboratory can thus be examined by this method of culture with every anticipation of meaningful results. The use of the technique in survey work in uncovering symptomless urinary tract infections has obvious advantages, particularly in simplicity of operation and convenience in transport.

The inclusion of para-nitrophenyl glycerol in the medium by preventing the spread of Proteus spp. allows a single medium to be used in contrast to the two media employed in the slide method of Naylor and Guttmann (1967). The reagent has been found not to interfere with the serological specificity or other characteristics of a variety of pathogenic microorganisms (Mackay-Scollay, 1968). The shelf life of the unit is indefinite. With the use of antibioticimpregnated paper strips laid along the inoculated medium, an indication of antibiotic sensitivity is provided at the time of direct culture and does not interfere with the enumeration of colonies on the remaining surface of the medium.

The colour changes effected in the medium on incubation, together with the sensitivity pattern and colonial characterization, enable tentative identification of the microorganisms responsible for a urinary tract infection, provided that the bacteria are present as the sole causative species; even so, it is frequently possible to distinguish the presence of two pathogens in the cultures obtained.

\section{REFERENCES}

Asscher, A. W., Sussman, M., Waters, W. E., Harvard Davis, R. H., and Chick, S. (1966). Lancet, 2, 1037.

Cattell, W. R., and Lefford, M. J. (1963). Brit, med. J., 1, 97.

Hoeprich, P. D. (1960.) J. Lab. clin. Med., 56, 899.

Kass, E. H. (1956). Trans. Ass. Am. Phycns, 69, 56.

Lancet (1968). 1, 732.

Leigh, D. A., and Williams, J. D. (1964). J. clin. Path., 17, 498

Mackay-Scollay, E. M. (1968). Proc. Aus. Soc. Microbiol., p. 17.

Mackey, J. P., and Sandys, G. H. (1965). Brit. med. J., 2, 1286.

Mackie, T. J., and McCartney, J. E. (1960). In Handbook of Bacteriology, edited by R. Cruickshank, 10th ed. p. 303. Livingstone, Edinburgh.

Naylor, G. R. E., and Guttmann, D. (1967). J. Hyg. (Lond.) 65, 367. 